\title{
Polarization Insensitive One-to-Six WDM Multicasting in a Silicon Nanowire
}

Pu, Minhao; Hu, Hao; Peucheret, Christophe; Ji, Hua; Galili, Michael; Oxenløwe, Leif Katsuo; Jeppesen, Palle; Hvam, Jørn Märcher; Yvind, Kresten

Published in:

ACP Technical Digest

Publication date:

2012

Link back to DTU Orbit

Citation (APA):

Pu, M., Hu, H., Peucheret, C., Ji, H., Galili, M., Oxenløwe, L. K., Jeppesen, P., Hvam, J. M., \& Yvind, K. (2012). Polarization Insensitive One-to-Six WDM Multicasting in a Silicon Nanowire. In ACP Technical Digest (pp. ATh4B.7). Optical Society of America.

\section{General rights}

Copyright and moral rights for the publications made accessible in the public portal are retained by the authors and/or other copyright owners and it is a condition of accessing publications that users recognise and abide by the legal requirements associated with these rights.

- Users may download and print one copy of any publication from the public portal for the purpose of private study or research.

- You may not further distribute the material or use it for any profit-making activity or commercial gain

- You may freely distribute the URL identifying the publication in the public portal

If you believe that this document breaches copyright please contact us providing details, and we will remove access to the work immediately and investigate your claim. 


\title{
Polarization Insensitive One-to-Six WDM Multicasting in a Silicon Nanowire
}

\author{
M. Pu, H. Hu, C. Peucheret, H. Ji, M. Galili, L. K. Oxenløwe, P. Jeppesen, J. M. Hvam, and K. Yvind \\ DTU Fotonik, Technical University of Denmark, Building 343, DK-2800 Lyngby, Denmark \\ mipu@fotonik.dtu.dk
}

\begin{abstract}
We present polarization insensitive one-to-six WDM multicasting based on nondegenerate four-wave mixing in a silicon nanowire with angled-pump scheme. Bit-error rate measurements are performed and error-free operation is achieved.

OCIS codes: (190.4380) Nonlinear optics, four-wave mixing; (190.4390) Nonlinear optics, integrated optics; (060.4255) Networks, multicast; (130.7405) Wavelength conversion devices.
\end{abstract}

\section{Introduction}

Multicasting is an important feature in a communication network, which can efficiently send a stream of information from a single source to multiple destinations [1], [2]. By using multicasting technology, the amount of network resources can be significantly reduced compared to unicast only networks. Optical layer multicasting could be implemented at a fixed wavelength by power splitting. An alternative is optical wavelength division multiplexing (WDM) multicasting, i.e., multicasting to different wavelengths. One of the most addressed techniques for WDM multicasting is all-optical multi-wavelength conversion (MWC), which replicates signals from one wavelength on multiple wavelengths. WDM multicasting has been demonstrated in different devices including semiconductor optical amplifiers (SOAs) [3], electroabsorption modulators (EAMs) [4], and highly nonlinear fibers (HNLFs) [5], [6], based on different nonlinear effects such as self-phase modulation (SPM), cross-phase modulation (XPM), cross-absorption modulation (XAM), and four-wave-mixing (FWM).

Recently, wavelength conversion in silicon nanowires has attracted considerable research interest due to its compactness, large conversion bandwidth [7] and complementary metal-oxide-semiconductor (CMOS) compatibility. Silicon nanowires have been utilized to demonstrate wavelength conversion at data rates from $10 \mathrm{~Gb} / \mathrm{s}$ [8] to $640 \mathrm{~Gb} / \mathrm{s}$ [9], and a sixteen-way multicasting operation of $40 \mathrm{~Gb} / \mathrm{s}$ non return-to-zero (NRZ) data has also been demonstrated in silicon nanowires [10]. However, sixteen continuous wave (CW) tunable laser sources (TLSs) are needed to act as input signals and phase information is not preserved in the converted signals. Previously, we have demonstrated one-to-six multicasting operation based on dual-pump FWM in a silicon nanowire [11]. The FWM process is normally highly polarization dependent and an efficient FWM process only occurs when the input signal and pump waves are both aligned to either transverse-electric (TE) mode or transverse-magnetic (TM) mode. However, a polarization insensitive operation is desired since the state of polarization (SOP) of the input signal fluctuates with time and distance in a real transmission system.

In this paper, we report polarization-insensitive one-to-six WDM multicasting of $10 \mathrm{~Gb} / \mathrm{s}$ differential phaseshift-keying (DPSK) data based on dual-pump FWM in a silicon nanowire using an angled-pump scheme [12]. Error-free performance is achieved for all six multicast signals while the polarization of the input data signal is continuously scrambled.

\section{Experiment}

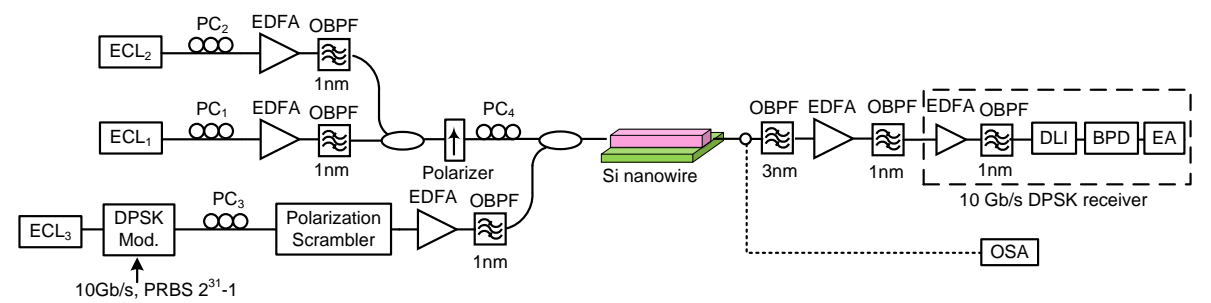

Fig. 1. Experimental setup for polarization-insensitive wavelength multicasting of $10 \mathrm{~Gb} / \mathrm{s}$ DPSK data in a silicon nanowire.

The experimental setup for the polarization-insensitive one-to-six WDM multicasting of a $10 \mathrm{~Gb} / \mathrm{s}$ DPSK signal in a silicon nanowire is shown in Fig. 1. The pump waves are generated from external cavity lasers $\left(\mathrm{ECL}_{1}\right.$ and $\left.\mathrm{ECL}_{2}\right)$ at $1545.7 \mathrm{~nm}$ and $1547.8 \mathrm{~nm}$, respectively. The signal wave, generated by ECL $\mathrm{EL}_{3}$ at $1554.5 \mathrm{~nm}$, is externally modulated by a Mach-Zehnder modulator (MZM) with a 10-Gb/s DPSK signal, encoded with a pseudo-random binary sequence (PRBS) of length $2^{31}-1$. All the pump and signal waves are amplified by erbium-doped fiber amplifiers 
(EDFAs), filtered by 1-nm optical band pass filters (OBPFs), and then combined by 3-dB couplers before being coupled into the silicon waveguide. The silicon nanowire is $1 \mathrm{~cm}$ long and its cross-sectional dimension is $\left(\mathrm{H} \times \mathrm{W}=300 \times 450 \mathrm{~nm}^{2}\right)$. It was fabricated on silicon-on-insulator (SOI) material using electron-beam lithography followed by reactive-ion etching. The silicon nanowire was inversely tapered at both ends and covered by a polymer waveguide for efficient coupling [13]. Two tapered fibers are used for coupling light into and out of the silicon waveguide. The polarization controller (PC) is used to adjust the polarization angle for the pump. At the output of the silicon waveguide, the converted signal (idler wave) is filtered out by two OBPFs, amplified by an EDFA in between, and detected by the 10-Gb/s DPSK receiver (shown by the dashed box in Fig. 1). In the receiver, the 10$\mathrm{Gb} / \mathrm{s}$ DPSK data signal is pre-amplified, filtered with a 1-nm OBPF and then demodulated by a one-symbol delay line interferometer (DLI). The output of the DLI is detected by a balanced photodetector (BPD), followed by a 10$\mathrm{Gb} / \mathrm{s}$ error analyzer (EA) for BER measurements. In order to test the polarization sensitivity, the SOP of the signal is continuously scrambled over the entire Poincaré sphere with a frequency $\sim 113 \mathrm{kHz}$. The polarization angle of the co-polarized pumps is adjusted to minimize the power variation of one of the converted idlers at the output of the silicon nanowire while the input signal polarization is scrambled.

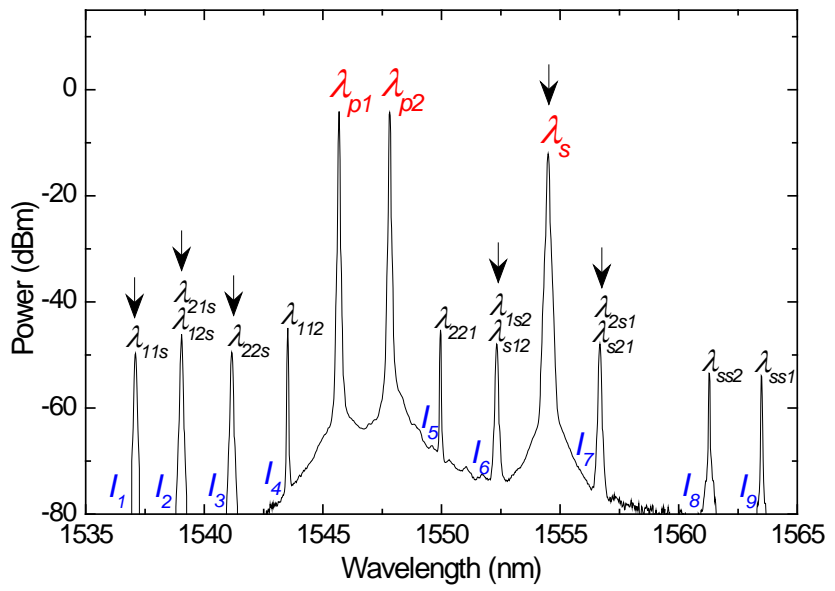

Fig. 2. Measured optical spectrum at the output of the silicon nanowire. The phase-preserved converted idlers are indicated by arrows.

Fig. 2 shows the spectrum measured at the output of the silicon nanowire. The wavelengths of two co-polarized CW pumps and the input signal are denoted $\lambda_{p 1}, \lambda_{p 2}$, and $\lambda_{s}$, respectively. The input powers of the two pumps and the signal are $18.4 \mathrm{dBm}, 18.9 \mathrm{dBm}$, and $9.4 \mathrm{dBm}$, respectively. Due to the dual-pump FWM process, nine idlers are generated as shown in Fig. 2. However, not all the converted idlers carry the same phase information as the original DPSK data. Here, we denote each FWM generated wave as $\lambda_{i j k}$, where the subscripts " $i$ " and " $j$ " refer to the two pump waves and " $k$ " refers to the signal wave in the corresponding FWM process. The phase information of the generated wave can be expressed as $\left(\Phi_{i}+\Phi_{j}-\Phi_{k}\right)$. To preserve the phase information of the original DPSK data in a generated FWM wave, there should be and can only be one wave that carries the original DPSK data $\left(\lambda_{s}\right)$ in the three wave components $\left(\lambda_{i}, \lambda_{j}, \lambda_{k}\right)$ of each FWM process. There are six output signals (including the output signal at $\lambda_{s}$ ), indicated by the arrows in Fig. 2, which satisfy the mentioned phase preserving requirement and thus can be decoded, resulting in the same phase information as the original DPSK data [11]. Therefore, one-to-six multicasting of the DPSK signal is achieved.

To characterize the performance of the polarization-insensitive WDM multicasting, we measured the BER of the $10 \mathrm{~Gb} / \mathrm{s}$ back-to-back and the multicast $10 \mathrm{~Gb} / \mathrm{s}$ DPSK data signals without and with the input signal polarization scrambled, as shown in Fig. 3. Error-free operation was obtained for all the multicast signals. It is also seen that there is no error floor for all the converted idler signals. The maximum power penalty of the wavelength multicast signals is $8 \mathrm{~dB}$ at $10^{-9}$ for the converted idler $I_{1}$. The relatively large power penalty is mainly due to the residual CW pumps and low optical signal-to-noise ratio (OSNR) induced by the large amplified spontaneous emission (ASE) noise from the EDFA. In addition, the relatively low conversion efficiency for idler $I_{1}$ also contributes to the relatively large power penalty. Besides, the additional power penalties caused by the polarization scrambling of the signal are smaller than $2 \mathrm{~dB}$. Fig. 3 (lower) shows the eye diagrams of the converted $10 \mathrm{~Gb} / \mathrm{s}$ DPSK data signals without and with the input signal being polarization-scrambled. It is seen that all the converted idler signals have clear and open eyes which are comfortably error free. The receiver sensitivity of the converted idler signals could be improved by better filtering away the residual CW pump or increasing the pump power, which will result in an increased OSNR and increased conversion efficiency. 


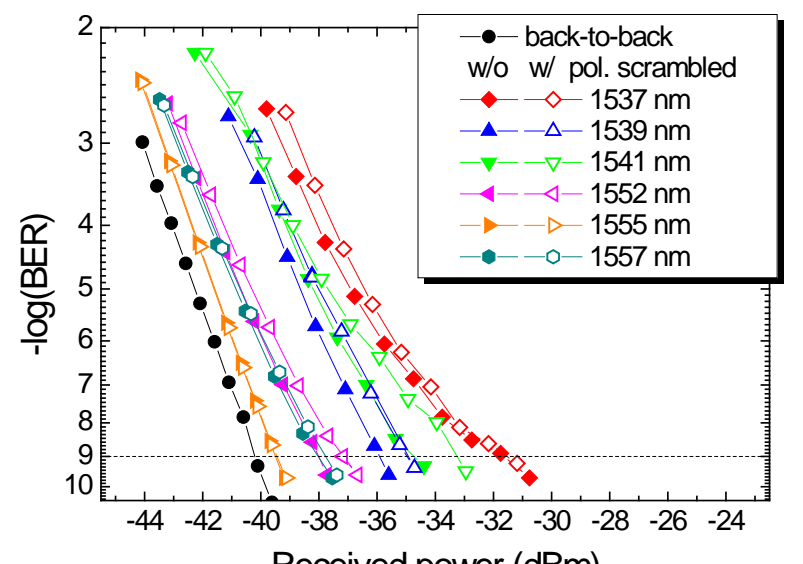

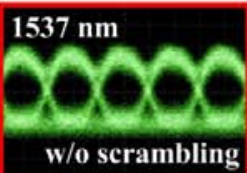
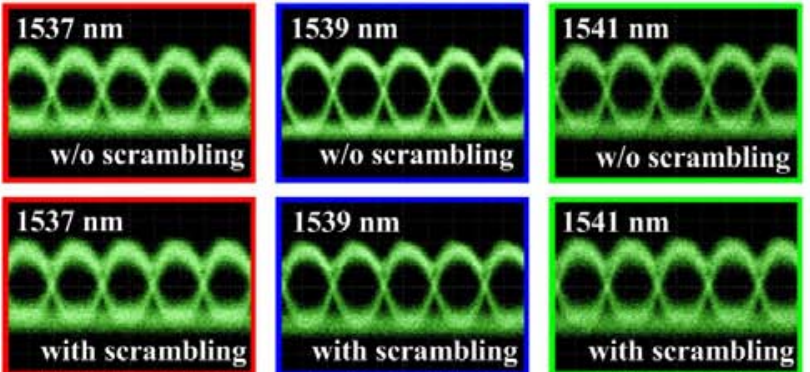
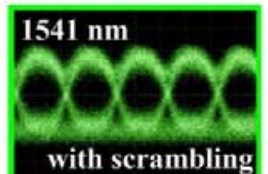
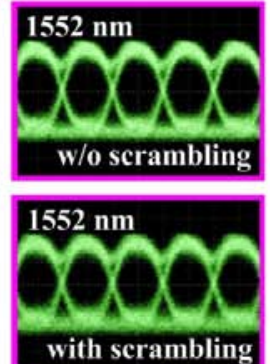
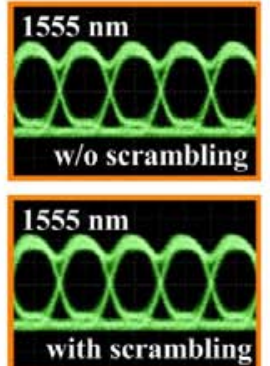
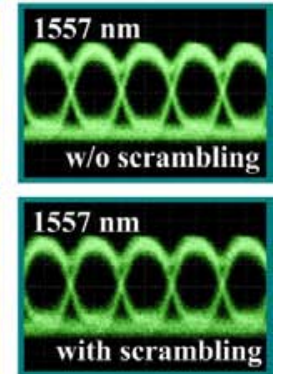

Fig. 3. (Upper) BER measurement for the $10 \mathrm{~Gb} / \mathrm{s}$ back-to-back DPSK signal, and for the multicast $10 \mathrm{~Gb} / \mathrm{s}$ DPSK signals without and with the input signal polarization scrambled. (Lower) Eye-diagrams of the multicast $10 \mathrm{~Gb} / \mathrm{s}$ DPSK signals with and without the input signal polarization scrambled.

\section{Conclusion}

We have experimentally demonstrated polarization insensitive one-to-six WDM multicasting of $10 \mathrm{~Gb} / \mathrm{s}$ DPSK data in a 1-cm long silicon nanowire based on dual-pump FWM. The polarization insensitive operation is realized with an angled-pump scheme. The phase information of the input DPSK signal has been successfully preserved and delivered from one wavelength to six multicast wavelengths using two pumps while the input signal polarization is continuously scrambled. In the BER measurement, all the multicast signals show error-free performance. The silicon nanowire could be useful for WDM multicasting applications in future WDM networks.

\section{Reference}

[1] X. Zhang, J. Wei, and C. Qiao, "Constrained multicast routing in WDM networks with sparse light splitting," J. Lightwave Technol., 18, Dec. 2000, pp. 1917-1927.

[2] R. K. Pankaj, “Wavlength requirements for multicasting in all-optical networks,” IEEE/ACM Trans. Networking 7, Jun. 1999, pp. 414-424.

[3] G. Contestabile, M. Presi, and E. Ciaramella, "Multiple wavelength conversion for WDM multicasting by FWM in an SOA," IEEE Photon. Technol. Lett., 16, Jul. 2004, pp. 1775-1777.

[4] L. Xu, N. Chi, K. Yvind, L. Christiansen, L. K. Oxenløwe, J. Mørk, P. Jeppesen, and J. Hanberg, “7×40 Gb/s base-rate RZ all-optical broadcasting utilizing an electroabsorption modulator,” Opt. Express, 12, pp. 416-420.

[5] G. Lu, K. S. Abedin, and T. Miyazaki, "DPSK multicast using multiple-pump FWM in Bismuths highly nonlinear fiber with high multicast efficiency,” Opt. Express, 16, Dec. 2008, pp. 21964-21970.

[6] C.S. Brès, A. O. J. Wiberg, B. P. P. Kuo, N. Alic, and S. Radic, "Wavelength multicasting of 320-Gb/s channel in self-seeded parametric amplifier,” IEEE Photon. Technol. Lett., 21, Jul. 2009, pp. 1002-1005.

[7] A. C. Turner-Foster, M. A. Foster, R. Salem, A. L. Gaeta, and M. Lipson, "Frequency conversion over two-thirds of an octave in silicon nanowaveguides," Opt. Express, 18, Feb. 2010, pp. 1904-1908.

[8] H. Rong, Y. H. Kuo, A. Liu and M. Paniccia, and O. cohen, "High efficiency wavelength conversion of $10 \mathrm{~Gb} / \mathrm{s}$ data in silicon waveguides,” Opt. Express, 14, Feb. 2006, pp. 1182-1188.

[9] H. Hu, H. Ji, M. Galili, M. Pu H. C. H. Mulvad, L. K. Oxenløwe, K. Yvind, J. M. Hvam, and P. Jeppesen, "Ultra-high-speed wavelength conversion in a silicon photonic chip,” Opt. Express, 19, Oct. 2011, pp. 19886-19894.

[10] A. Biberman, B. G. Lee, A. C. Turner-Foster, M. A. Foster, M. Lipson, A. L. Gaeta, and K. Bergman, "Wavelength multicasting in silicon photonic nanowires," Opt. Express, 18, Aug. 2010, pp. 18047-18054.

[11] M. Pu, H. Hu, H. Ji, M. Galili, L. K. Oxenløwe, P. Jeppesen, J. M. Hvam, and K. Yvind, "One-to-six WDM multicasting of DPSK signals based on dual pump four-wave mixing in a silicon waveguide,” Opt. Express, 19, Nov. 2011, pp. 24448-24453.

[12] S. Gao, X. Zhang, Z. Li, and S. He, "Polarization-Independent Wavelength conversion using an angled-polarization pump in a silicon nanowire waveguide,’IEEE J. Sel. Topics Quantum Electron. 16, Jan. 2010, pp. 250-256.

[13] M. Pu, L. Liu, H. Ou, K. Yvind, and J. M. Hvam, “Ultra-low-loss inverted taper coupler for silicon-on-insulator ridge waveguide,” Opt. Commun., 283, Oct. 2010, pp. 3678-3682. 